\title{
SURVEY OF SAFETY PRACTICES AMONG HOSPITAL LABORATORIES IN OROMIA REGIONAL STATE, ETHIOPIA
}

\author{
Tsegaye Sewunet ${ }^{1}$, Wakjira Kebede ${ }^{1}$, Beyene Wondafrash ${ }^{2}$, Bereket \\ Workalemau ${ }^{3}$, Gemeda Abebe ${ }^{2}$
}

\begin{abstract}
BACKGROUND: Unsafe working practices, working environments, disposable waste products, and chemicals in clinical laboratories contribute to infectious and non-infectious hazards. Staffs, the community, and patients are less safe. Furthermore, such practices compromise the quality of laboratory services. We conducted a study to describe safety practices in public hospital laboratories of Oromia Regional State, Ethiopia.

METHOD: Randomly selected ten public hospital laboratories in Oromia Regional State were studied from Oct 2011- Feb 2012. Self-administered structured questionnaire and observation checklists were used for data collection. The respondents were heads of the laboratories, senior technicians, and safety officers. The questionnaire addressed biosafety label, microbial hazards, chemical hazards, physical/mechanical hazards, personal protective equipment, first aid kits and waste disposal system. The data was analyzed using descriptive analysis with SPSS version16 statistical software.

RESULT: All of the respondents reported none of the hospital laboratories were labeled with the appropriate safety label and safety symbols. These respondents also reported they may contain organisms grouped under risk group IV in the absence of microbiological safety cabinets. Overall, the respondents reported that there were poor safety regulations or standards in their laboratories. There were higher risks of microbial, chemical and physical/mechanical hazards.

CONCLUSION: Laboratory safety in public hospitals of Oromia Regional State is below the standard. The laboratory workers are at high risk of combined physical, chemical and microbial hazards. Prompt recognition of the problem and immediate action is mandatory to ensure safe working environment in health laboratories.

KEYWORDS: laboratory safety, district hospital, Oromia Region
\end{abstract}

DOI: http://dx.doi.org/10.4314/ejhs.v24i4.5

\section{INTRODUCTION}

Health institutions are a place where most exposure to clinical biohazard exists. Particularly, those which deal with clinical specimens such as laboratories, have high exposure to contamination and risk of infection. Exposure to non-infectious hazards such as cut, skin injuries, electric shock, fire, explosion and burns with corrosive chemicals and poisoning with toxic substances are also common $(1,2)$. A range of activities are carried out in laboratories which include the handling of infectious patient samples with a visible microbial hazard to the professional and to the patient. Other hazards or safety problems may arise with in the laboratory itself from built in physical agents like different instruments, electrical establishments, and potential toxic chemicals routinely used in the laboratory procedures on daily basis (3).

\footnotetext{
${ }^{1}$ Depertement of Microbiology, Jimma University, Ethiopia

${ }^{2}$ Department of Medical Laboratory Sciences and Pathology, Jimma University, Ethiopia

${ }^{3}$ Department of Laboratory Technology, Arbaminch College of Health Sciences, Ethiopia

Corresponding Author: Tsegaye Sewnet, Email: tsegishs2010@gmail.com
} 
According to the World Health Organization, the health laboratory should have minimum standard to be fulfilled depending on the level and the scope of the laboratory (4). Specimen reception room and procedure working areas should be separable from office and considered as potentially infected areas and they should have biohazard symbol; floors should be slip resistant and impermeable to liquids. The surface walls and partitions should be smooth, impervious and easily cleanable. One or more of these rooms should be designed for reception, treatment and disposal of contaminated waste. There should be sufficient ventilation and light, and in case of accident, there should be some mechanism to cope with it. Practically, most laboratory operations are hampered because of limited space or insufficient use of the space available. This is because insufficient attention has been given to designing and planning of laboratory structure (5).

WHO reported around $80 \%$ of the total waste generated by health laboratories in developing countries is infectious and not decontaminated; the disposal systems in these institutions are not well organized. In Ethiopia, only $26.1 \%$ of laboratories dispose wastes after decontaminating; the rest either do not have waste disposal system or they do not dispose according to standard procedure (6).

The reliability of the laboratory results which may determine the fate of patients, the community, and also clinical management of cases in general is dependent on the safety and quality level of these laboratories. Hence, determining the safety level of the health laboratories scaling the biosafety level depending on the range of activities covered by individual laboratories is mandatory. It also helps to indicate where to start if rearrangement or restructuring of the laboratory safety issues are inevitable.

\section{METHODS}

A cross sectional study was conducted among ten randomly selected (lottery method) public hospital laboratories from Oct 2011-February 2012 in Oromia Region, Ethiopia. Oromia Regional state is the largest of the nine regions in Ethiopia. According to the 2008 census, the region is reported to have a population of about 28.8 million. Of these approximately $85 \%$ live in rural areas. This state is administratively divided into 17 zones and 5 towns and has 31 hospitals according to 2000 (EFY). Variables like biosafety label, microbial hazards, chemical hazards, physical/mechanical hazards, personal protective equipment, first aid kits and waste disposal system were studied. Selfadministered structured questionnaire and observation checklist were prepared. After pretest the questionnaire and observation checklist were used for data collection and the data was analyzed through descriptive statistics using statistical software (SPSS ver.16).

The study was conducted after ethical clearance was obtained from Jimma University, College of Public Health and Medical Sciences institutional review board, and the participating laboratories consented to participate in the study.

\section{RESULTS}

General condition of the laboratory: A total of 10 laboratories out of 31 public hospital laboratories in Oromia Regional State were randomly selected and included in the study. Seventy percent of the respondents were heads of the laboratories $20 \%$ senior technicians and $10 \%$ safety officers. One safety officer and eight of the other respondents have bachelor of sciences degree in medical laboratory sciences. The number of technical staffs working in these hospitals ranges from 4-22 (an average of 9 technicians). The number of patients visiting these hospitals ranges from 40-350 per day. Ninety percent of the respondents reported that the hospitals have sufficient space for work. Nearly all (80\%) of these respondents reported that their labs were not labeled with appropriate biosafety label. Only $30 \%$ of the hospital laboratories reported that there they had safety officers. None of the hospital labs have safety guidelines to follow in the laboratory as identified during observation by the investigators.

No accident books were available in the laboratories, only 30\% reported they have both safety officer and safety guideline. With regard to storage of chemicals, $50 \%$ reported the labs have enough space for storage of chemicals and reagents. All laboratories reported that there is no lined budget or expenditure for the laboratory safety.

Microbial hazard: All laboratories reported that they contain organisms classified as risk group II to IV in their laboratories. Some (30\%) of the laboratories have functional autoclave and use it for sterilization, whereas $70 \%$ of the laboratories either have no autoclave or it is not functional; instead, they use different techniques including boiling, dry heat, $70 \%$ alcohol, hypochlorite solution, soap and water depending on availability. Five of the laboratories also transport specimen between units of the laboratory and with other laboratories, but they did not follow any kind of specimen transportation standard.

Waste from the laboratory was disposed by the cleaners to incinerator in all laboratories; however, there was no standard procedure followed about the disposal of laboratory waste. The cleaners from all the laboratories have completed at least junior secondary school; yet' did not attend any formal training pertaining to the cleaning, handling and disposal of waste in general. The water supply of the laboratories was also reported to vary between laboratories, $40 \%$ reported the supply was intermittent; whereas $60 \%$ of the laboratories do not think the water is pure.

Hand washing practice was also assessed; only $30 \%$ of the respondents reported they wash their hands always before leaving the laboratory and after each test analysis; 70\% reported they wash their hands sometimes in the laboratory and/or between tests. All of them reported they use soap and water for hand washing; none of them reported use of antiseptics. In general, there is no guideline followed for microbial agents handling and disposal in all the laboratories.

Chemical hazard: Most of the laboratories (60\%), reported they have a list of records of chemicals and reagents; however, not all the chemicals are labeled with full information that describes the chemical. Only $30 \%$ of the laboratories responded the chemicals used in the labs have safety symbols labeled clearly. The labels were reportedly given by the manufacturers, by technicians in the lab and others they don't know who labeled the labels.

Expired chemicals were also contained in these labs and disposals of these expired chemicals may take more than six months. Some labs $(40 \%)$ reported that chemicals are disposed to incinerator including volatile and flammable chemical; $60 \%$ of the laboratories do not know where chemicals, reagents and left overs/expired items or spills are disposed. 
Overall, perceived risk of chemical hazard is understood as explosion of fire from chemicals. They mentioned careful handling of chemicals as prevention and reduction of the risk, but none of the labs specified what careful handling is about. Only one laboratory reported to have functional fire extinguisher.

Physical and mechanical hazard: The working benches of the laboratories were rated to be overcrowded by $70 \%$ of the laboratories based on their own professional judgment; the rest responded that there was sufficient space for work. The electrical voltage was set by the electricians as reported by all the labs, but they do not know whether the power is above optimum or not. The electrical equipment in $70 \%$ of the labs do not have instruction manual with them for the reason they do not know; either not supplied with the instrument or lost within the labs. Most, $70 \%$ of the labs do not have maintenance schedule for instruments for the reason they do not know. Other labs that reported to have maintenance schedule informed that the maintenance schedule is determined based on the need; there were no evidence of maintenance log book or scheduled maintenance.

Broken glass wares also used in $30 \%$ of the labs were studied for the reason there is shortage of glass wares. In the rest of the labs, broken glasses are disposed by throwing in to dust bin, putting them back to mini store, or else, cleaners dispose them to waste disposal pits.

The corridors were reported to be open, no blockade to corridors and emergency exits. There were no nonfunctional equipment and/or instruments placed in the lab. The overall observation of these labs and response of the labs indicate the labs have no sufficient space for work and there might be risk of mechanical or physical hazard.

Personal safety measures

Respondents were asked about personal protective, equipment and instruments, the availability of laboratory coats, gloves, masks, eye shields, safety cabinets and usage. The respondents from $50 \%$ of the laboratories reported they do not have sufficient laboratory coats individually, and they wear the available coats some times and may be not at all. Face masks were reported not available in $80 \%$ of the laboratories; only $20 \%$ of laboratories have and they wear it sometimes. There is no eye shield or goggle reported in any of the labs.

Availability of clean gloves is reported from $90 \%$ of the laboratories; only $40 \%$ of the labs reported they use it always during specimen handling, processing and disposal of waste from the laboratories. Most $(60 \%)$ of the laboratory personnel wear gloves sometimes or may not wear at all even in the presence of gloves. Biosafety cabinets were reported available only in 30\% of the laboratories; all of them do not know the level of containment of the biosafety cabinet. These laboratories do not know how to use it and what the level is and specifically what is handled in these biosafety cabinets.

First aid and accident handling: Laboratory first aid kit and first aid log book are not available in any of the laboratories. Most of them reported they do not know the reason. Only 30\% of respondents reported lack of attention from the administration. None of the respondents had training on first aid. Needle injury accidents were reported in $30 \%$ of the laboratories, and one reported to the emergency clinic for a help, one reported to ART clinic for post exposure prophylaxis and the other left unattended.
Finally, all the labs suggested that first aid kits should be available. Training should be given for at least two laboratory personnel from each lab. Safety practices should be strengthened nationally.

\section{DISCUSSION}

This survey addressed important and wider range of laboratory safety issues in the randomly selected public hospital laboratories from the largest regional state of Ethiopia: Oromia Region. The survey addressed main areas of safety concerns in medical laboratories: physical environment of the working place, microbiological safety, chemical safety, waste disposal system and safe working practice among the workers. There is limited or no study available to show the picture of clinical laboratories safety issues in Ethiopia, particularity in Oromia Region. This study is a prototype providing baseline insight on main safety concerns in the studied laboratories and can be used to estimate the safety status of other laboratories in Ethiopia.

Clinical laboratories are expected to be safe and operate under maximum safety standards to protect their workers, the community and the patients they serve (7). Safe working practice is also related to the reliability of laboratory results. However, laboratories in poor settings are suffering from poor safety practices, and the results of the laboratories that we studied are not an exception $(8,9)$. Although the laboratories reported differently, based on observation by investigators, the most commonly identified weaknesses with regard to safety parameters include: lack of sufficient space in most laboratories $(80 \%)$, absence of safety guide lines, lack of safety officer (90\%), absence of accident book, and lack of lined budget for safety. As strengths, the corridors were reported to be open; there were no blockades to corridors and emergency exits. The overall observation of these labs and the responses of the labs indicate the labs have no sufficient space for work, and there might be risk of biological, chemical and mechanical or physical hazards. The findings of this study show that these laboratories were below the standard set by WHO, Good Clinical Laboratory Practice (GCLP) (10).

International standards required that it is the level of the laboratory that determines the type of microorganism to be processed (11). However, all laboratories in our study reported that they encountered organisms up to risk level IV regardless of their laboratory level. Assessments of their microbiological safety was very poor: with only $30 \%$ labs having functional autoclave and most use another means of "sterilization"; none of the laboratories follow any kind of specimen transport standard; all lab wastes were incinerated by untrained cleaners without any written standard and poor hand washing practice. It is also reported in Ethiopia that the medical laboratories with standard biological safety cabinet (safe exhaust outlet) do not exceed $33 \%$ (12).

Although there were lists of chemical records in all laboratories assessed, all chemicals were not labeled with full chemical information and it is unknown who labels some of the chemicals. Expired chemicals were also found on the shelves of all labs and the disposal system is either delayed for more than 6 months or expired chemicals are disposed to incinerator with no regard to the nature of the chemical including volatile and flammable chemicals. In addition, most $(60 \%)$ of the labs do not know where chemicals, reagents and leftovers or 
spills were disposed. This situation is particularly dangerous from the view of providing accurate laboratory results as it is very difficult to determine the right chemical and reagents to be used for the lab tests. Moreover, the high likelihood of using expired reagents because of inappropriate labeling compromises the reliability of lab results. Poor handling of chemicals in terms of storage and disposal pose a particular risk to the worker and the community (13).

Electrical standards and equipment management system were far below the reported standards, and are considered as the primary cause of physical and mechanical hazards. These include crowded working bench (70\%), lack of knowledge about output voltage of their electrical system, lack of instruction manual for electrical equipment (70\%), and lack of equipment maintenance schedule (70\%). Broken glass wares are also used in three of the labs studied and disposal of broken glasses is reported to be thrown into dust bin, put it back to mini store, or else, cleaners dispose it to waste disposal pits (14).

Personal safety measures indicate that the availability and/or usage of personal protective equipment (PPE) were very poor or nil (laboratory coats, gloves, masks, eye shields), and biosafety cabinets were available only in $30 \%$ of the laboratories even though the personnel do not know the level of containment, how to use the biosafety cabinet and what is handled in them (15). Laboratory first aid kit and first aid log book were not available at all in any of the laboratories and none of the lab personnel in the laboratory has had training on first aid. Needle injury accidents were reported in $30 \%$ of the laboratories, and one of them reported to the emergency clinic, one of them reported to ART clinic and the other left unattended; there was a similar report from another study (2). In our study, short supply of PPE was reported. Whenever available, usage of PPE is not according to the recommended practice (16). Another previous study from Ethiopia on HIV screening laboratories also revealed only $29 \%$ them follow safety guidelines (17).

Accident management and first aid were reported not known by any of the laboratories. These clearly indicate the risk and hazard of these laboratories to the laboratory personnel, patients and the hospital community (18).

In general, the laboratory workers are at high risk of combined physical, chemical and microbial hazards. Prompt recognition of the problem and immediate action is mandatory to ensure safe working environment in health laboratories. Furthermore, these laboratories may be potential threats to the hospital environment and risk the safety and care of patients at large. On the other hand, to see the bigger picture in the country, national and large scale study should be conducted.

\section{ACKNOWLEDGEMENT}

We are thank full to all participating laboratories and Jimma University, College of Public Health and Medical Sciences, Research and Post graduate Coordinating Office for financial support.

\section{REFERENCES}

1. Cheesbrough M, District laboratory practice in Tropical Countries. Part 1, 2005: Cambridge University Press.

2. Chelenyane M, R. Endacott. Self-reported infection control practices and perceptions of
HIV/AIDS risk amongst emergency department nurses in Botswana. Accid Emerg Nurs, 2006. 14(3):148-54.

3. Vonesch N, Tomao P, Di Renzi S, Vita S, Signorini S. Biosafety in laboratories concerning exposure to biological agents. $G$ Ital Med Lav Ergon, 2006. 28(4)444-56.

4. WHO, District health laboratory safety guideline for developing countries. 2002:26-29.

5. WHO, Laboratory biosafety manual. 3 ed. 2004, Geneva.

6. Regulatory world santacorolaa country, Stanford's laboratory safety manual-April 2002.

http://www.stanford.edu/dept/ehs/prod/aboutus/ documents/safetymanual. accessed on08/09/2013.

7. Delhi, I.C.o.M.R.N., Gude lines for Good Clinical Laboratory Practices (GCLP) http://icmr.nic.in/guidelines/GCLP.pdf, 2008. accessed on $08 / 22 / 2013$.

8. Ogunnowo B. E, Anunobi C. C, Onajole A. T, Odeyemi K. A. Awareness of occupational health hazards and the practice of universal safety precautions among mortuary workers in South West. Nig Q J Hosp Med, 2011. 20(4):192-6.

9. Fadeyi A, Fowotade A, Abiodun M. O, Jimoh A. K, Nwabuisi C, Desalu O. O, Awareness and practice of safety precautions among healthcare workers in the laboratories of two public health facilities in Nigeria. Niger Postgrad Med J, 2011. 18(2):141-6.

10. WHO, Good Clinical Laboratory Practice (GCLP/08).http://www.who.int/tdr/publications /documents/gclp-web.pdf, 2009. accesses on $01 / 04 / 2014$

11. 11. Harrington $J$ and Shannon S, Harrington J. Shannon S. Survey of safety and health care in British medical laboratories. British Medical Journal, 1977. 1:626-628.

12. Fantaw S. Performance assessment of clinical microbiology laboratories in Ethiopia; bacterial identification and antibiotic susceptibility testing. http://epaethiopia.org/book-ofabstract/item/25-performance-assessment-ofclinical-microbiology-laboratories-in-ethiopiabacterial-identification-and-antibioticsusceptibility-testing. 10/17/2013.

13. Joke K, Kolhater B. Laboratory safety precaution. 3 ed. 2003, Philadelphia: J.B. Lippincott.

14. Cutter J. and Gammon J. Review of standard precautions and sharps management in the community. $\mathrm{Br} J$ Community Nurs, 2007. 12(2):54-60.

15. Hinkin, J., J. Gammon, and J. Cutter, Review of personal protection equipment used in practice. Br J Community Nurs, 2008. 13(1):14-9.

16. FMoHE, Disease prevention and control Department. Infection prevention guidelines for health care facilities in Ethiopia. 2004.

17. Belete T, Hailu M, Wegene T, et al., The status of HIV screening laboratoriesin Ethiopia: achievments, problems encountered,and possible solution. Ethiop. J. Health Dev., 2002. 16(2): 209-215.

18. CDC, Guidelines for Biosafety Laboratory Competency. MMWR, 2011. 60. 